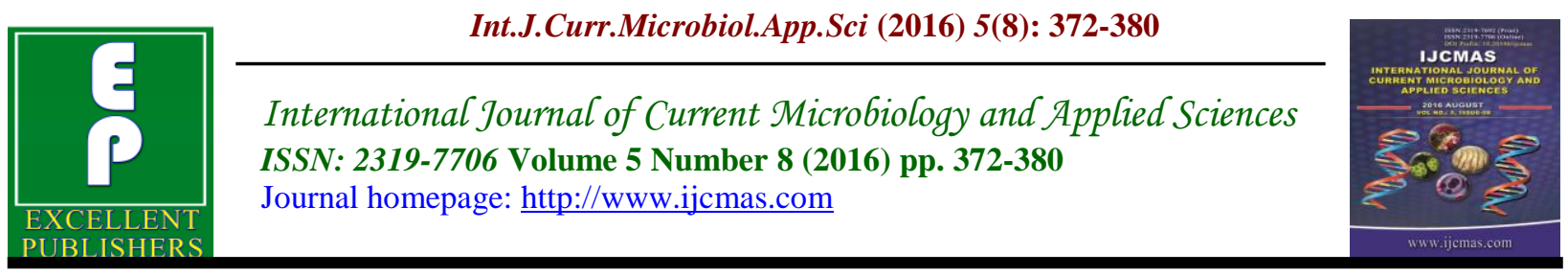

Original Research Article

http://dx.doi.org/10.20546/ijcmas.2016.508.039

\title{
Incidence and Culture Characteristics of Dermatophytes Isolated at a Tertiary Care Hospital in the Outskirts of Hyderabad, India
}

\author{
R. Saraswati Jayanthi, R. Nagamani*, M. Lavanya and Raghavendra Rao \\ Malla Reddy Medical College for Women Suraram, Hyderabad -55, India \\ *Corresponding author
}

Keywords

Dermatophytes,

Trichophyton

rubrum,

Microsporum

species.

\begin{tabular}{l}
\hline Article Info \\
\hline Accepted: \\
17 July 2016 \\
Available Online: \\
10 August 2016
\end{tabular}

\section{A B S T R A C T}

Dermatophytosis, affecting skin, nails, and hair, is the commonestclinical condition encountered in the dermatology clinics in the tropical countries. Laboratories plays an important role in confirming the diagnosis when the clinical picture is not very clear. Good practical knowledge of fungal culture techniques and identification methods are crucial for better isolation ratesand accuracy of diagnosis. The present study involved60 patients with suspected dermatophyte infection attending Dermatology, Venereology and Leprosy clinic in Malla Reddy Narayana Multispecialty hospital, Suraram, Hyderabad.Fungal elements were seen in 20 cases by direct potassium hydroxide $(\mathrm{KOH})$ mount examination and dermatophytes grew in 17 out of these 20cases. The overall positivity rate was $33 \%$. The predominant infectionobserved in this study was tinea corporis and the predominant fungus isolated was Trichophyton rubrum. Among the dermatophytes, Trichophyton rubrum grew rapidly ( $\leq 20$ days), the earliest being within in 7 days. Microsporum species were comparatively slow growing, all three isolates grew after 20 days. In spite of being a very common condition in our country not much has been done about control of this disease. Most laboratories don't isolate or have sufficient clinical data on susceptibility pattern of these fungal infections.

\section{Introduction}

Superficial fungal infections of the skin is public health problem (Abdelkarim et al., 2013, Judith, 2006). Dermatophytosis which involves keranized tissues such as superficial skin, nails and hair, is the commonest condition that is encountered in the dermatology clinics (Lakshmi et al., 2015). These infections are usually ignored and patient presents to outpatient department only if cosmetically disfiguring or due to inconvenience caused by pruritus. This results in many not seeking medical help immediately thus increasing the disease load in the community and increased risk of transmission. The epidemiology of dermatophytosis has evolved over the last many decades. Whether developed countries or developing countries these changes have occurred due to changing life styles, economy, increasing leisure activities and environmental factors. People who are exposed to soil, and animals occupationally or as a leisure activity, are at risk of acquiring dermatophyte infection. 
Immunocompromised individuals are another group who are at risk (Rebecca et $a l$., 2012). In addition to the information about host risk factors, the knowledge of fungal pathogenic mechanisms helps in understanding the disease and treating appropriately.

Prevalence of site of infection varies with geographic area and patient groups. Tinea pedis and onycomycoses are more common in developed countries and tinea corporis, tinea cruris and tinea capitis in developing countries (Rebecca, 2012). Tinea capitis typically occurs in children due to physiological and hormonal factors. Though the commonly occurring type of infection is Tinea corporis followed by Tinea cruris, different studies described Tinea unguim (Reena Roy et al., 2014; Gupta et al.,2014), hair infection (Anup Kainthola et al.,2014, Rashmi et al., 2014) as commonest conditions.

Increase in the contact with pet animals may result in increasing incidence of dermatophytosis of zoophilic origin. Different dermatophyte species exhibit varying degrees of pathogenicity. Anthropophilic fungi produce chronic infection, while zoophilic and anthropophilic spp produce more inflammatory lesions. In vitro studies with co cultures of keritinocytes and Trichophyton mentagrophytes, a zoophilic species, showed there are high levels of IL8, and $\mathrm{TNF} \alpha$ production. Simultaneous expression of proteases and survival from phagocytosis also contribute to the inflammatory response (Judith, 2006).

In general, these infections are confined to keratinized surfaces like skin, nail, and hair, but they can produce other types of clinical diseases, such as hypersensitivity. Studies have shown that there is association between
Trichophyton infection and hypersensitivity (Arunaloke et al., 2014; Judith, et al., 2006). Microsporum species one of the zoophilic fungi is reported to have caused invasive infection in HIV patients (King et al., 199).

The patients attending this hospital are mostly from the surrounding villages. The aim of this study is to find the fungal agents causing superficial mycoses with special reference to dermatophytes in rural population who are more at risk due to their life style and occupation.

\section{Materials and Methods}

60 patients attending Dermatology and Venereology clinic in Malla Reddy Narayana Multispeciality Hospital, Suraram with suspected dermatophytic infection were included in the present study.

The study was conducted at department of Microbiology in the mycology section of service labs. Skin scrapings/hair/nail clippings were collected by the dermatologist after cleaning the area with alcohol and into sterile butter paper. Specimens were then sent to the microbiology laboratory for processing. Patient details like age, sex, occupation, physical activity, diagnosis, site of lesion, treatment were also collected.

\section{Processing of the specimen}

All samples were subjected to microscopy and culture

Microscopy: Potassium hydroxide preparation (KOH): Few drops of $15 \% \mathrm{KOH}$ solution were placed on the slide and a portion of the specimen added to this. A cover slip was placed over the $\mathrm{KOH}$ mount, warmed over the flame and left for 15 minutes for clearing of specimen. In case 
of nail, it is left for longer period till the cells are dissolved. The slide was examined for the presence of fungal elements.

Culture: Sabouraud's dextrose agar (SDA) with gentamicin (8mg/litre)were used for culturing the specimens initially. Later dermatophyte test medium(DTM) was also included for culturing the specimens. All cultures were incubated at $25^{\circ} \mathrm{C}$ in biochemical oxygen demand incuators/B.O.D incubator for 4 weeks before issuing a negative report. Plates and tubes were examined everyday during first week and every two days thereafter. This was done to detect any bacterial or saprophytic fungal contamination and also to re-inoculate in case contamination occurred. The day of the appearance of the suspected dermatophyte colony was noted for assessing the rate of growth of the isolates.

\section{Identification of the isolates}

Rate of growth, colony morphology, pigment on obverse and reverse were noted. Lactophenol cotton blue mount were prepared from the suspected colonies and examined microscopically. Presence of any conidia, type, size, shape were noted, and any other special structures like spiral hyphae, or racquet hyphae were also looked for. Identification of the dermatophyte was according to the standard procedure.

Where necessary, slide cultures and urease test were performed.

A portion of the specimen was saved for reexamination or re-inoculation when necessary.

If any suspected dermatophyte colony was mixed with contaminating colonies, the colony was sub-cultured again on SDA with Gentamicin or on DTM.

\section{Results and Discussion}

60 patients comprising 44 males and 16 females were included in this study.Predominant infection observed was tinea corporis.The distribution of the cases by site are shown in Table no. 1, and figure no. 1 .

Table.1 Distribution of cases by infection site

\begin{tabular}{|l|l|l|l|l|l|l|l|l|}
\hline Site & $\begin{array}{l}\text { T.corpori } \\
\mathrm{s}\end{array}$ & $\begin{array}{l}\text { T.cruri } \\
\mathrm{s}\end{array}$ & T.facei & $\begin{array}{l}\text { T.manu } \\
\mathrm{um}\end{array}$ & $\begin{array}{l}\text { T.pedi } \\
\mathrm{s}\end{array}$ & $\begin{array}{l}\text { t.ungui } \\
\mathrm{m}\end{array}$ & $\begin{array}{l}\text { T.capit } \\
\text { is }\end{array}$ & $\begin{array}{l}\text { T.barba } \\
\mathrm{e}\end{array}$ \\
\hline No.(\%) & $42(66.6)$ & $5(8.7)$ & $3(5.2)$ & $3(5.2)$ & $4(7)$ & $4(7)$ & $5(5.2)$ & $1(1.75)$ \\
\hline
\end{tabular}

* 5 cases have more than one site of infection.

Table.2 Results of $\mathrm{KOH}$ and culture

\begin{tabular}{|l|l|l|l|}
\hline & KOH positive & KOH negative & TOTAL \\
\hline CULTURE positive & 12 & 5 & $\mathbf{1 7}$ \\
\hline CULTURE negative & 8 & 35 & $\mathbf{4 3}$ \\
\hline TOTAL & $\mathbf{2 0}$ & $\mathbf{4 0}$ & $\mathbf{6 0}$ \\
\hline
\end{tabular}


Table.3

\begin{tabular}{|l|c|c|c|c|c|c|}
\hline Site & T.rubrum & T.mentagrophyte & T.tonsurans & Epidermophyton & Microsporum & Candida \\
\hline T.corporis & 6 & 3 & 1 & 1 & 2 & 1 \\
\hline T. cruris & & & & & - & - \\
\hline T.capitis & 2 & - & - & & & - \\
\hline T.pedis & 1 & & & 1 & 3 & 2 \\
\hline Total & 9 & 3 & 1 & & \\
\hline
\end{tabular}

Table.4 Rate of growth of isolates

\begin{tabular}{|l|l|l|l|l|l|l|}
\hline Days & T.rubrum & T.mentagrophytes & T.tonsurans & $\begin{array}{l}\text { Epidermophyton } \\
\text { spp }\end{array}$ & $\begin{array}{l}\text { Microsporum } \\
\text { spp }\end{array}$ & Total \\
\hline Within 7days & 1 & - & - & - & - & \\
\hline 8-14 days & 5 & 3 & 1 & 1 & - & 1 \\
\hline 15-21days & 3 & - & - & - & - & 10 \\
\hline $\begin{array}{l}\text { More than } \\
\text { 21days }\end{array}$ & - & - & - & - & 3 & 3 \\
\hline Total & 9 & 3 & 1 & 1 & 3 & 3 \\
\hline
\end{tabular}

Fig.1 Distribution of cases by site of infection



T.C-tinea corporis, T.CR -tinea cruris, T.F- tinea facei, T.M- tinea manum, T.U -tinea unguim, T.CP- tinea capititis, T.B- tinea barbae 
Fig.2 Microsporum gypseum colony on reverse and obverse

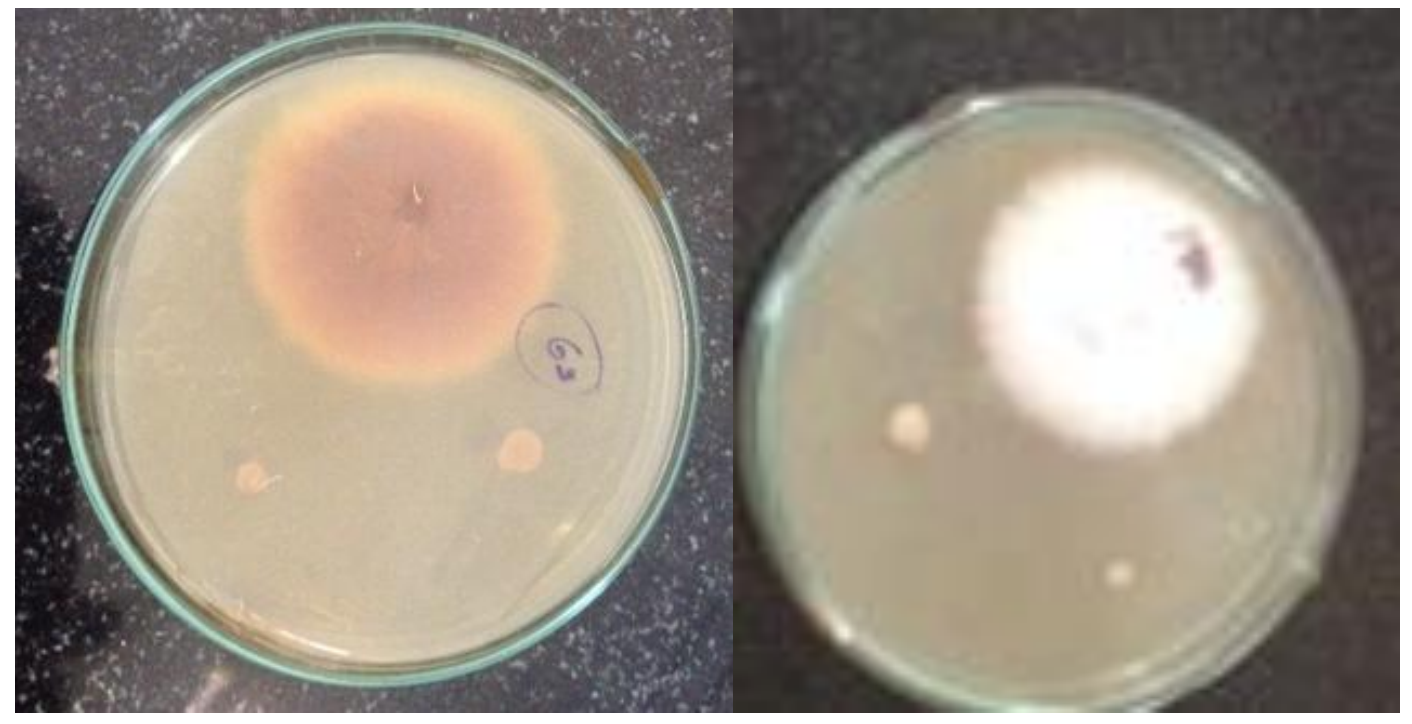

Fig.3 Colony variant of T.rubrum on Dermatophyte test medium

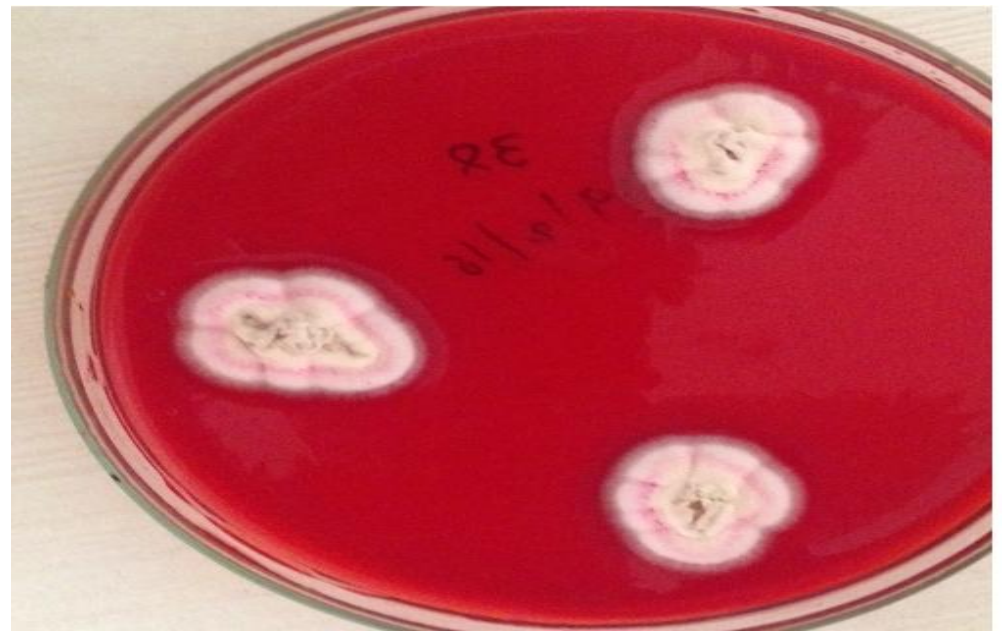

Trichophyton rubrum colony obverse and reverse side

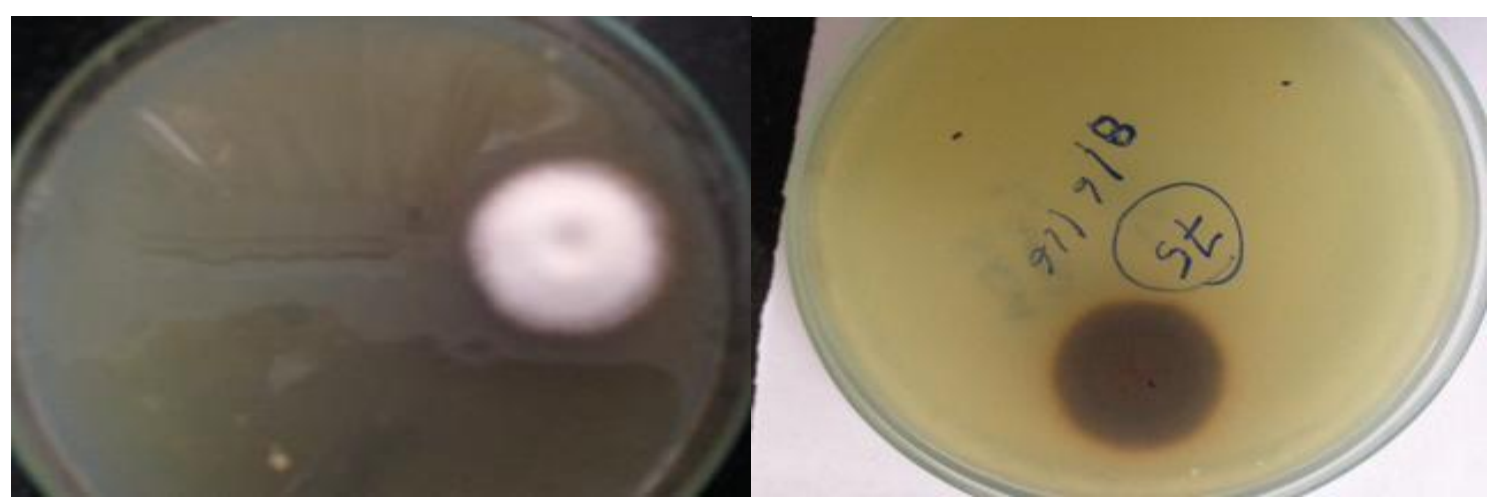


Fig.4 Lactophenol cotton blue preparation showing spiral hyphae of T. mentagrophytes

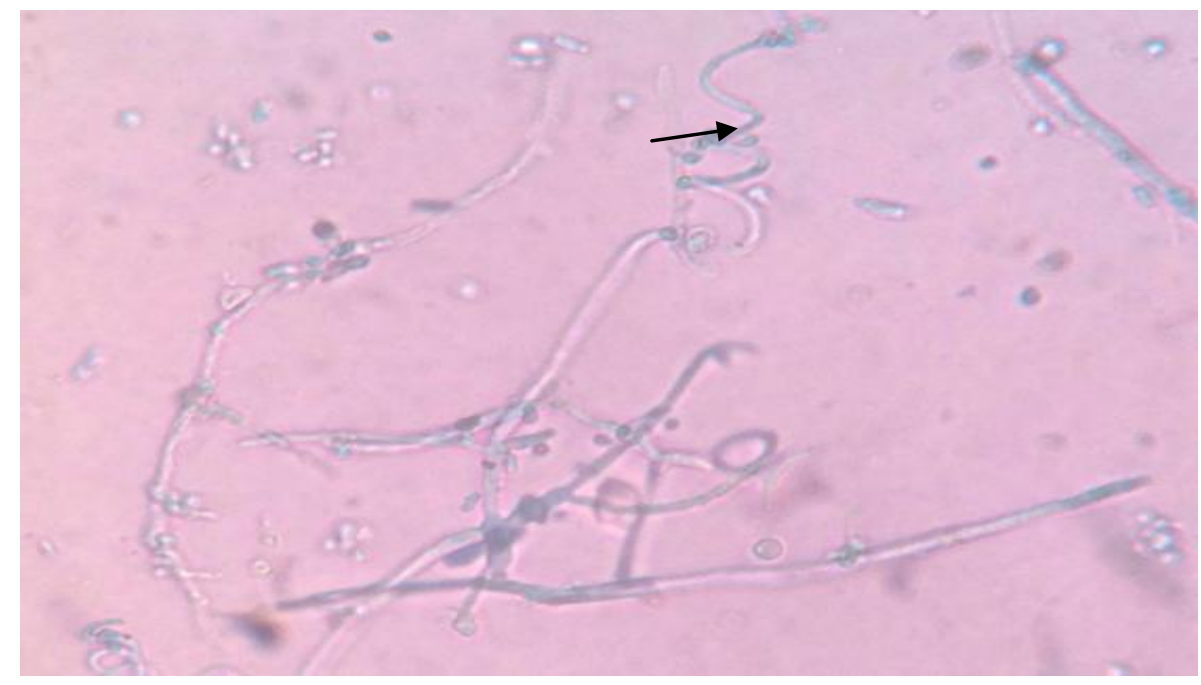

Fig.5 Lactophenol cotton blue preparation showing Pencil shaped macroconidia in T.rubrum

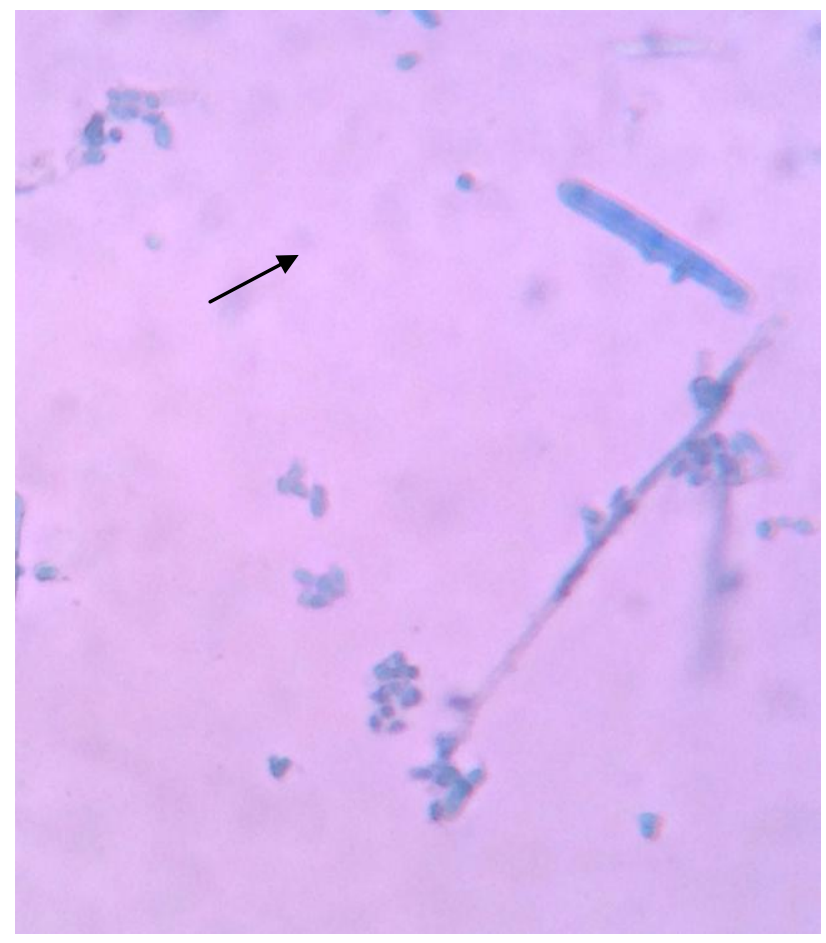

The overall positive rate either in $\mathrm{KOH}$ examination or culture is $41.6 \%$. Distribution of the cases which were $\mathrm{KOH}$ positive or negative, culture positive, or negative is shown in Table no. 2 . Predominant type of dermatophyte isolated belonged to the genus Trichophyton. The incidence of different fungal isolates is given in Table no. 3. Coinfection with Candida spp. was seen in three cases, two of which were isolated from Tinea pedis cases and one was isolated along with $T$. rubrum from a case of Tinea corporis. One of the isolates of Trichophyton rubrum exhibited 
colony variation showing a cottony type of growth with a pink colored concentric ring on the surface (Fig no.3). The dermatophyte that showed rapid growth was $T$. rubrum (7days) from a case of tinea corporis. The isolates which took longer time were Microsporum spp (more than 20days). Single isolate of Epidermophyton species from a case of Tinea corporis showed growth in 14days. The average rate of growth was 14.5 days. Details of the rate of growth of different isolates is given in table no. 4.

The occurrence of dermatophytes in different geographic areas is different because of the differences in climate, topographic differences, and social customs. In India, Trichophyton spp are predominant causative agents of superficial skin infections irrespective of the topography. Trichophyton rubrum was found to be the predominant fungus in studies from different parts of India, (Doddamani et al., 2013; Lakshmi et al., 2015; Kennedy kumar et al., 2007; Madhavi et al., 2011; Suman singh et al., 2003; Smita Sharma et al., 2007; Kannan et al., 2006) as in our study though not the site of infection. On the other hand, Microsporum canis is important pathogenic fungus in middleeastern countries like Saudi Arabia, Kuwait (Abanmi et al., Razzaq Adel et al., 2007). Nevertheless Trichophyton spp follow Microsporum species in these countries also. In African countries like Nigeria (Kennedy et al., 2015), Egypt (Abdelkarim, et al., 2013) the infecting agents are similar to that of Indian subcontinent.

Though our study involved less number of cases, the positive results are equivalent to other studies. Various studies show culture positivity is more than $\mathrm{KOH}$ positivity and vice versa. Our study gave higher number of $\mathrm{KOH}$ positive cases. In case of $\mathrm{KOH}$ positive and culture negative instances, it can be explained by the fact that all of them were seen in the beginning of the study when specimens were processed by technicians who were not adequately trained in the fungal culture techniques. Another explanation is probable absence of fungal elements in the cultured portion of the specimen. $\mathrm{KOH}$ negative and culture positive cases can also be due to same reasons as well as the efficiency of the examining microbiologist. With available basic media and scarcely trained technicians, our attempts yielded fairly good results.

Addition of DTM improved isolation rates but not turnaround times as DTM is not satisfactory for identification of the fungus (Sowmya et al., 2014). In spite of all these pitfalls, the cultures could be identified within 2-3 weeks. Microsporum spp grew very slow and also not profusely. On the other hand T.rubrum and T. mentagrophytes grew abundantly. The single isolate of Epidermophyton species did not show any special property in the growth rate.

In conclusion, the incidence and the fungal agents causing dermatophytosis is similar to other studies and patient group may influence the site of infection and the fungi causing them.

This study shows that good technical skills and in depth knowledge in mycology are of paramount importance for improving the isolation and identification rates in addition to good specimen collection methods. In view of the public health importance, patients may be encouraged to seek medical help in dermatology clinics during health camps.

\section{References}

Abanmi, A., Bakheshwain, S., El Khazzi, N. et al., 2008. characteristics of superficial fungal infections in the 
Riyadh region of Saudi Arabia. Int. J. Dermatol., 47: 229-235

Abdelkarim Mahmoud Aboueisha, Heba El Mahallawy. 2013 Public health significance of Dermatophytes Ismailia, Port Said, Egypt. Med. Mycol. J., vol.54, 123-129.

Anup Kanthiola, Puneet Gaur, Alkesh Dobhal, Shailendra. 2014. Int. Rese. J. Med. Sci., vol 2(8) 9-12.

Arunloke Chakrabarti, Subhash Chandra Sharma. Association of hypersensitivity and carriage of dermatophytes in clinically normal sites in patients with tinea cruris. Mycopathologia, vol.37, issue 2, pg 71-74.

King, D., L.W. Cheever, A. Hood, T.D. Horn, M.G. Rinaldi. 1996. Primary invasive cutaneous Microsporum canis infection in immunocompromised patients. $J$. Clin. Microbiol., p460-462.

Gupta, C.M., Tripathi, K., Tiwari, S., Rathore, Y., Nema, S., Dhanvijay, A.G. 2014. Current trends of clinicomycological profile of dermatophytosis in central India. $J$. Dent. Med. Sci., vol.13, p23-26.

Judith, A., Woodfolk. 2006. Allergy and dermatophytes. Clin. Microbiol. Rev., p30-43.

Kennedy Kumar, Anupma Jyothi Kindo, J., Kalyani, S., Anandan. 2007. Clinicomycological profile of dermatophytic skin infections in a tertiary care center - a cross sectional study. Sri Ramachandra $J$. med., vol 1, issue 2, Jan.

Kennady, T., Warso, Jeremia, A., Igunma, Ibinado, L., Oboro. 2015. Patterns of dermatophytes isolated in medical microbiology laboratory of the University of Port Harcourt Teaching Hospital, Riverside, Nigeria. Adv. Microbiol., 5; 346-350.
Lakshmi Vasantha poluri, Jyothi, P., Indugula, Sai, L., Kondapaneni. 2015. Clinicomycological study of dermatophytosis in south india. $J$. Lab Physicians, 7: 84-9.

Madhavi, S., Ramarao, M.V., Jyothsna, K. 2011. Mycological study of dermatophytosis in rural population. Annals of Biol. Res., 2(3): 88-93.

Kannan, P., C. Janaki, G.S. Selvi. 2006. Prevalence of dermatophytes and other fungal agents isolated from clinical specimens. I.J.MM, 24(3): $212-5$

Doddamani, P.V., K.H. Harshan, R.C. Kanta, R. Gangane, K.B. Suni. 2013. Isolation, Identification, and prevalence of dermatophytes in tertiary care hospital in Gulbarga district. Peoples J. Scientific Res., vol.6(2).

Razzaq Adel, A.A., Sultan, A.O., Basmiah, A.M. et al., 2007. Prevalence of tinea capitis in Southern Kuwait. Mycoses, 50: 317-320.

Rebecca Rashid Achterman, Theodore, C., white. 2012. Dermatophyte virulence factors: Identifying and analyzing genes that may contribute to chronic or acute skin infections. Int $J$. Microbiol., vol. pg 1-8.

Rebecca Rashid Achterman, Theodore, C., White. 2012. A foot in the door for dermatophyte research. PLoS Pathogens, march, vol.8, issue 3

Reena Roy Ghosh, Rathindranath Ray, Tamalkanti Ghosh. 2014. Clinicomycological profile of Dermatophytosis in a tertiary care hospital in West Bengal. Int. J. Of Curr. Microbiol. Appl. Sci., Vol.3, no.9, pg 655-666.

Rashmi, P., P Mahale, M. Raghavendra rao, Tejashree, A., Deepashri, Madhuri Kulkarni. 2014. Clinicomycological profile of dermatophytosis in a 
teaching hospital. Int J. Pharma. Sci. Invention, vol.3(8): 43-46.

Smita Sharma, A.K., Borthakur. 2007. A clinico epidemiological study of dermatophytoses in north east India. I.J.DVL, Nov-Dec, vol73, issue 6.

Soumya Nasimuddin, Appalaraju, B, Surendran, P, Srinivas, C.R. 2004. Isolation, identification and comparative analysisof SDA, and DTM for dermatophytes from clinical samples in a tertiary care hospital. IOSR JDMS, Vol.13,11: Nov.

Suman Singh, P.M., Beena. 2003. Profile of dermatophyte infections in Baroda. Indian J. Dermatol. Venerol. Leprol., vol. 69, issue 4.

\section{How to cite this article:}

Saraswati Jayanthi, R., R. Nagamani, M. Lavanya and Raghavendra Rao. 2016. Incidence and Culture Characteristics of Dermatophytes Isolated at a Tertiary Care Hospital in the Outskirts of Hyderabad, India. Int.J.Curr.Microbiol.App.Sci. 5(8): 372-380. doi: http://dx.doi.org/10.20546/ijcmas.2016.508.039 